\title{
METALLICITY EFFECTS ON THE CEPHEID DISTANCE SCALE
}

\author{
WENDY L. FREEDMAN \\ Observatories of the Carnegie Institution of Washington \\ Pasadena Calif. 99101 \\ USA \\ BARRY F. MADORE \\ NASA/IPAC Extragalactic Database \\ UPL/Caltech \\ Pasadena Calif. 91125 \\ $U S A$
}

\begin{abstract}
We are undertaking empirical tests for the effects of metallicity on the zero-point of the Period-Luminosity relations for Cepheids in M31 and in the Small Magellanic Cloud (SMC), as compared to the Large Magellanic Cloud (LMC). For M31 we have used BVRI data on comparable sets of Cepheids in three radially separated, and chemically different, fields in order to solve simultaneously for the reddenings and the true distance moduli to each of these fields. Recent theory predicts that the reddeningcorrected moduli should differ by $0.8 \mathrm{mag}$ given the observed metallicity range in our M31 Cepheid sample; we observe a maximum range of only $0.17 \mathrm{mag}$. For the SMC BVRIJHK data, treated in a similar fashion, the predicted theoretical effects on the short wavelength (B and V) data may be visible, but with low statistical certainty and at a reduced amplitude with respect to the predictions.
\end{abstract}

\section{Introduction}

Gascoigne (1974) was the first person to attempt a comprehensive evaluation of the effects of metallicity on the observed colours and magnitudes of Cepheids. His motivation was the known differences in metallicity between the Population I components of LMC, SMC and the Galaxy; his concern was that line blanketing in combination with structural differences induced by metallicity variations could plausibly affect the observed properties of Cepheids to the extent that corrections to the Cepheid distance scale would become necessary. From a purely theoretical point of view Stothers (1988) again reviewed the situation and concluded that metallicity effects on the Cepheid distance scale should be appreciable especially if the Cepheids are self-consistently corrected for reddening using $(\mathrm{B}, \mathrm{V})$ data. Recently we have begun applying multi-wavelength fitting techniques (Freedman 1988) to readdress this problem from a purely empirical point of view.

As discussed in detail in Freedman and Madore (1990), M31 provides the first test site for this metallicity effect. From the extensive work of Baade and Swope in the 1960s, Cepheids are available in fields whose mean metallicities range from 1.7 to 0.3 times solar, as indicated by independent studies of the radial gradient of metallicity in HII regions and supernova remnants. Accordingly, we have observed three sets of Cepheids at radial distances of 3,10 and $20 \mathrm{kpc}$ from the centre of M31, spanning a metallicity range of approximately five. Using the prime focus CCD at the CFHT we have obtained complete BVRI light curves for nearly 40 Cepheids from which intensity-mean magnitudes and colours have been derived. Scaled against the LMC Cepheid data, we have been able to derive differential apparent distance moduli for each of these three fields in 
each of the four bandpasses. From these observations (see Figure 1) we find little evidence for any significant departure of the data from a fit to a simple differential Galactic extinction law, while the zero points in the fits (the inferred true moduli) are consistent with the sense of the deviations predicted by theory but with a much reduced (a factor of four lower) absolute metallicity effect.

We have applied the same multi-wavelength fitting technique to the published LMC/SMC $B V R I J H K$ data. A simple Galactic extinction law again provides a very good fit to the seven wavelengths, well within the uncertainties of the individual distance determinations. Small (less than one-sigma) deviations for the $B$ and $V$ data are present in the same sense as predicted by theory, but with a reduced amplitude (as also found for M31). Details will be presented elsewhere (Freedman and Madore 1990, in preparation).

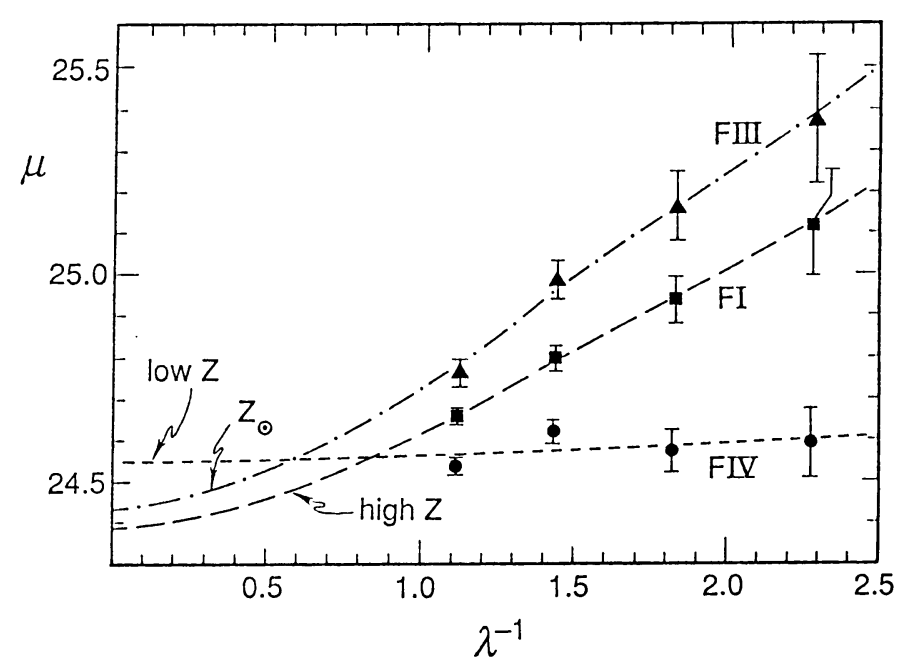

Figure 1. Apparent modulus as a function of inverse wavelength for our M31 Cepheid data. The fits are to a standard Galactic extinction law, where the intercept is the true modulus. Field I (at $3 \mathrm{kpc}$ from the centre of M31) is high metallicity, and moderate reddening; Field III (at $10 \mathrm{kpc}$ ) is of solar metallicity and has the highest reddening; while Field IV (at $20 \mathrm{kpc}$ ) is of both low metallicity and low reddening.

\section{References}

Freedman, W.L. (1988), Astrophys. J. 326, 691.

Freedman, W.L. and Madore, B.F. (1990), Astrophys. J. in press.

Gascoigne, S.C.B. (1974), M.N.R.A.S. 166, 25P.

Stothers, R.B. (1988), Astrophys. J. 329, 712. 